\section{Philadelphia-like acute lymphoblastic leukemia is associated with minimal residual disease persistence and poor outcome. First report of the minimal residual disease-oriented GIMEMA LAL1913}

\author{
Sabina Chiaretti, ${ }^{1 *}$ Monica Messina,,${ }^{1,2^{*}}$ Irene Della Starza,,${ }^{1,2}$ \\ Alfonso Piciocchi, ${ }^{2}$ Luciana Cafforio, ${ }^{1}$ Marzia Cavalli, ${ }^{1}$ Akram Taherinasab, ${ }^{1}$ \\ Michela Ansuinelli, ${ }^{1}$ Loredana Elia, ${ }^{1}$ Guglielmo Albertini Petroni, ${ }^{1}$ \\ Roberta La Starza, ${ }^{3}$ Martina Canichella, ${ }^{1}$ Alessia Lauretti, ${ }^{1}$ Maria Cristina \\ Puzzolo, ${ }^{1}$ Valentina Pierini, ${ }^{3}$ Alessandra Santoro, ${ }^{4}$ Orietta Spinelli, ${ }^{5}$ Valerio \\ Apicella, ${ }^{1}$ Saveria Capria, ${ }^{1}$ Francesco Di Raimondo, ${ }^{6}$ Paolo De Fabritiis, ${ }^{7}$ \\ Cristina Papayannidis, ${ }^{8}$ Anna Candoni, ${ }^{9}$ Roberto Cairoli, ${ }^{10}$ Marco Cerrano, ${ }^{11}$ \\ Nicola Fracchiolla, ${ }^{12}$ Daniele Mattei, ${ }^{13}$ Chiara Cattaneo,${ }^{14}$ Antonella Vitale, ${ }^{1}$ \\ Enrico Crea, ${ }^{2}$ Paola Fazi, ${ }^{2}$ Cristina Mecucci, ${ }^{3}$ Alessandro Rambaldi, ${ }^{5}$ \\ Anna Guarini, ${ }^{15}$ Renato Bassan ${ }^{16}$ and Robin Foà ${ }^{1}$
}

${ }^{1}$ Hematology Unit, Department of Translational and Precision Medicine, Sapienza University, Rome; ${ }^{2}$ GIMEMA Data Center, Fondazione GIMEMA Franco Mandelli Onlus, Rome; ${ }^{3}$ Hematology and Bone Marrow Transplantation Unit, Department of Medicine, University of Perugia, Perugia; ${ }^{4}$ Hematology and Bone Marrow Transplant Unit, Ospedali Riuniti Villa Sofia-Cervello, Palermo; ${ }^{5} \mathrm{Hematology}$ and Bone Marrow Transplant Unit, Ospedale Papa Giovanni XXIII, Bergamo; ${ }^{6}$ Haematology Unit, Department of General Surgery and Medical-Surgical Specialties, University of Catania, Catania; ${ }^{7}$ Hematology Unit, S. Eugenio Hospital, Rome; ${ }^{8}$ Seragnoli Institute of Hematology, Bologna University School of Medicine, Bologna; ${ }^{9} \mathrm{Clinica}$ di Ematologia e Unità di terapie Cellulari 'Carlo Melzi'-Azienda Sanitaria-Universitaria Integrata, Udine; ${ }^{10} \mathrm{Hematology}$ Unit, Niguarda Hospital, Milan; ${ }^{11} \mathrm{Hematology}$ Unit, Department of Oncology, Presidio Molinette, AOU Città della Salute e della Scienza di Torino, Torino; ${ }^{12} \mathrm{UOC}$ Oncoematologia, Fondazione IRCCS Ca' Granda Ospedale Maggiore Policlinico di Milano, Università degli Studi di Milano, Milan; ${ }^{13}$ Department of Hematology, Ospedale S. Croce, Cuneo; ${ }^{14}$ Department of Hematology, ASST Spedali Civili, Brescia; ${ }^{15}$ Department of Molecular Medicine, Sapienza University, Rome and ${ }^{16} \mathrm{Hematology}$ Unit, Ospedale dell'Angelo and Ospedale Ss Giovanni e Paolo, Mestre, Venezia, Italy

*SC and MM contributed equally as co-first authors.

\section{ABSTRACT}

T1 arly recognition of Philadelphia-like (Ph-like) acute lymphoblastic leukemia (ALL) cases could impact on the management and outcome of this subset of B-lineage ALL. In order to assess the prognostic value of the Ph-like status in a pediatric-inspired, minimal residual disease (MRD)driven trial, we screened $88 \mathrm{~B}$-lineage ALL cases negative for major fusion genes (BCR-ABL1, ETV6-RUNX1, TCF3-PBX1 and KTM2Ar) enrolled in the GIMEMA LAL1913 front-line protocol for adult $B C R / A B L 1$-negative ALL. The screening - performed using the "BCR/ABL1-like predictor" - identified $28 \mathrm{Ph}$-like cases (31.8\%), characterized by CRLF2 overexpression $(35.7 \%)$, JAK/STAT pathway mutations (33.3\%), IKZF1 (63.6\%), BTG1 (50\%) and EBF1 $(27.3 \%)$ deletions, and rearrangements targeting tyrosine kinases or CRLF2 (40\%). The correlation with outcome highlighted that: i) the complete remission rate was significantly lower in $\mathrm{Ph}$-like compared to non- $\mathrm{Ph}$ like cases $(74.1 \%$ vs. $91.5 \%, P=0.044)$; ii) at time point 2 , decisional for transplant allocation, $52.9 \%$ of Ph-like cases versus $20 \%$ of non-Ph-like were MRD-positive ( $P=0.025)$; iii) the $\mathrm{Ph}$-like profile was the only parameter associated with a higher risk of being MRD-positive at time point $2(P=0.014)$; iv) at 24 months, Ph-like patients had a significantly inferior event-free and disease-free survival compared to non-Ph-like patients (33.5\% vs. $66.2 \%$, $P=0.005$ and $45.5 \%$ vs. $72.3 \%, P=0.062$, respectively). This study documents
Haematologica 2021

Volume 106(6):1559-1568

Correspondence:

SABINA CHIARETTI

chiaretti@bce.uniroma1.it

Received: January 24, 2020.

Accepted: May 20, 2020.

Pre-published: May 28, 2020.

https://doi.org/10.3324/haematol.2020.247973

(C)2021 Ferrata Storti Foundation

Material published in Haematologica is covered by copyright. All rights are reserved to the Ferrata Storti Foundation. Use of published material is allowed under the following terms and conditions:

https://creativecommons.org/licenses/by-nc/4.0/legalcode. Copies of published material are allowed for personal or internal use. Sharing published material for non-commercial purposes is subject to the following conditions:

https://creativecommons. org//icenses/by-nc/4.0/legalcode, sect. 3. Reproducing and sharing published material for commercial purposes is not allowed without permission in writing from the publisher. 
that Ph-like patients have a lower complete remission rate, event-free survival and disease-free survival, as well as a greater MRD persistence also in a pediatric-oriented and MRD-driven adult ALL protocol, thus reinforcing that the early recognition of Ph-like ALL patients at diagnosis is crucial to refine risk-stratification and to optimize therapeutic strategies. Clinicaltrials gov. Identifier: 02067143.

\section{Introduction}

Philadelphia-like (Ph-like) acute lymphoblastic leukemia (ALL) accounts for $15-30 \%$ of B-lineage ALL, with an increasing incidence starting from adolescence. The growing interest in this subgroup of ALL arises from the distinctive gene expression profile - that resembles that of the true Ph-positive cases - and by the unfavorable clinical outcome. ${ }^{1,2}$ In-depth and large-scale genetic characterization has shown that the majority of Ph-like ALL cases carry fusion genes involving tyrosine kinases (i.e., ABLclass and JAK2 rearrangements), or cytokine receptor rearrangements (i.e., P2RY8/CRLF2 and IGH/CRLF2), frequently associated with mutations of the JAK/STAT pathway genes. ${ }^{3.5}$ Among the other co-operating events, a relevant role is played by IKZF1 deletions present in about $70 \%$ of cases. ${ }^{47}$ The possibility of recognizing these cases at diagnosis has important prognostic implications and would also pave the way to testing tyrosine kinase inhibitors (TKI) and other targeted therapeutic approaches that have proven successful in pre-clinical models and in vivo in a few relapsed patients., ${ }^{3,8-12}$ So far, several strategies $^{13-15}$ have been reported in an attempt to identify $\mathrm{Ph}$ like cases, but none of them is deemed as the gold standard for the diagnostic work-up of these patients. To this end, our group recently reported a predictive tool called "BCR/ABL1-like predictor" based on the levels of expression of nine genes together with CRLF2 transcript quantification. . From a clinical standpoint, Ph-like patients are characterized by a worse outcome which is due to an inferior response to induction therapy, a higher incidence of relapses and lower survival. ${ }^{1,2,4}$ Since minimal residual disease (MRD) is considered today the most important prognostic factor in ALL, the role of the Ph-like status has been investigated in the context of MRD-driven protocols, with contradicting results. Roberts and colleagues reported in a pediatric cohort that $\mathrm{Ph}$-like patients, though displaying higher MRD levels at the end of induction, had a survival probability similar to that of non-Ph-like childhood ALL when treated with intensive therapies. ${ }^{16}$ Opposite results were obtained by Heatley et al. ${ }^{14}$ who demonstrated that, despite a risk-adjusted treatment approach, a high rate of relapse was recorded among children who were retrospectively identified as Ph-like. In adolescents and young adults, the results of the CALGB10403 trial, based on a pediatric inspired regimen, have shown that parameters associated with inferior survival rates were indeed represented by the Ph-like signature and obesity. ${ }^{17}$ In adult cohorts, all reported studies so far agree on a shorter survival likelihood for Ph-like ALL compared to non-Ph-like patients. ${ }^{5.7,18,19}$ However, the data are still insufficient to elucidate whether intensive treatments are capable of abolishing the negative impact of the Ph-like status on prognosis: conflicting results have been reported in the studies by Jain et al..$^{20}$ and Herold et al. ${ }^{6}$ Likewise, the role of the Ph-like status in the context of MRD-driven clinical trials is still unclear, since the data produced by the
German study group were derived from a small cohort of patients. ${ }^{6}$

In order to clarify these aspects, we hereby evaluated the incidence and clinical-biologial features of $\mathrm{Ph}$-like cases - identified using the BCR/ABL1-like predictor - and the prognostic role of the $\mathrm{Ph}$-like profile in terms of complete remission (CR) achievement, MRD persistence and survival in a cohort of adult ALL patients homogeneously and intensively treated in the pediatric-oriented, MRDdriven LAL1913 GIMEMA front-line protocol for adult Phnegative ALL.

\section{Methods}

\section{Study population and experimental strategy}

This study included $\mathrm{B}$-lineage ALL patients negative for major molecular aberrations (BCR/ABL1, KT2MA and TCF3/PBX1, BNEG) enrolled in the GIMEMA LAL1913 front-line clinical trial (clinicaltrials gov. Identifier: 02067143; Online Supplementary Figure S1) - designed for Ph-negative ALL patients aged 18-65 years - based on a pediatric-oriented backbone, in which Pegasparaginase was administered instead of asparaginase, and on a MRD-driven transplant allocation; ${ }^{20} \mathrm{MRD}$ time-points and MRD analysis are detailed in the Online Supplementary Materials and Methods. The EC study number approval is 5629 .

Diagnostic bone marrow samples were available from 105 patients (median age 38.7 years, range, 18.2-64.7). Baseline patients' characteristics are summarized in the Online Supplementary Table S1; there were no differences in clinicalbiologial features between our cohort and the remaining population enrolled in the protocol (Online Supplementary Table S2). All cases underwent centralized molecular screening: i) the "BCR/ABL1-like predictor" assay, ii) sequencing of the JAK/STAT and RAS cascades by next-generation sequencing (NGS), iii) Multiplex Ligation-dependent Probe Amplification (MLPA), iv) targeted RNA sequencing. In 17 cases, the BCR/ABL1-like predictor was not feasible due to lack of RNA (Online Supplementary Table S3; Online Supplementary Figure S2).

\section{$B C R / A B L 1$-like predictor}

In order to detect the Ph-like cases, we applied the "BCR/ABL1-like predictor" to 88 patients (Online Supplementary Materials and Methods).

\section{Screening of recurrent mutations and deletions}

The members of the JAK/STAT (JAK1,JAK2,JAK3,IL7R and CRLF2) and RAS (FLT3, NRAS, KRAS and PTPN11) pathways (181 amplicons) were sequenced by NGS (Online Supplementary Materials and Methods).

NGS experiments were performed in 91 cases $(74$ in common with the BCR/ABL1-like predictor analysis - $24 \mathrm{Ph}$-like and 50 non-Ph-like ALL cases -, Online Supplementary Materials and Methods and Table 3). Variants recognized as single nucleotide polymorphisms (SNP) were excluded, unless of prognostic value or previously reported in Ph-like ALL. ${ }^{21}$

Recurrent deletions (IKZF1, CDKN2A/2B, PAX5, EBF1, BTG1, 
Table 1. Comparison between Philadelphia-like (Ph-like) and non-Ph-like clinical features.

\begin{tabular}{|c|c|c|c|}
\hline & Ph-Hike & non-Ph-ilke & $P$ \\
\hline $\mathrm{N}$ & 28 & 60 & \\
\hline Age, median (range) & $42.24(18.18-64.53)$ & $34.52(18.23-64.59)$ & ns \\
\hline Wbc $\times 10^{9} / L$, median (range) & $3.34(0.23-347)$ & $5.74(1-75.5)$ & ns \\
\hline Hb g/dL, median (range) & $8.70(3.70-13.00)$ & $9.75(5.00-15.70)$ & 0.034 \\
\hline Plt x10 $/ 4$, median (range) & $40(1.23-399)$ & $66.5(7.5-630)$ & ns \\
\hline \multicolumn{4}{|l|}{ Sex, N (\%) } \\
\hline M & $19(67.9 \%)$ & $34(56.7 \%)$ & ns \\
\hline $\mathrm{F}$ & $9(32.1 \%)$ & $26(43.3 \%)$ & \\
\hline \multicolumn{4}{|l|}{ Risk category, N (\%) } \\
\hline Standard risk & $14(56 \%)$ & $34(63 \%)$ & ns \\
\hline No Standard risk & $11(44 \%)$ & $20(37 \%)$ & \\
\hline
\end{tabular}

Ph-like: Philadelphia-like; N: number;WBC: while blood cell; Plt: platelet; M. male; F: female; ns: not significant.

RB1, ETV6 and CRLF2) were screened in 87 samples (70 in common with the BCR/ABL1-like predictor analysis - $22 \mathrm{Ph}$-like and 48 non-Ph-like ALL cases -, Online Supplementary Table S3), by the Salsa MLPA P335 ALL-IKZF1 kit (MRC-Holland, Amsterdam, the Netherlands) and analyzed according to the Coffalyser manual. ${ }^{22}$ P2RY8/CRLF2 was inferred when a deletion within the PAR1 region was documented. Samples were defined $I K Z F 1+C D K N 2 A / 2 B$ and/or PAX5 when IKZF1 deletion cooccurred with $C D K N 2 A / 2 B$ and/or PAX5 deletions. ${ }^{23}$

\section{Targeted RNA-sequencing and FISH analysis}

In order to detect fusion genes, libraries were prepared using the TruSight RNA Pan-Cancer Panel (Illumina, San Diego, CA) kit, targeting 1385 cancer- genes (Online Supplementary Materials and Methods).

Double-color fluorescence in situ hybridization (FISH) studies were performed in $20 \mathrm{~B}$-ALL, $13 \mathrm{Ph}$-like and seven non-Ph-like with high levels of CRLF2 expression (Online Supplementary Materials and Methods).

Overall, 85 cases were screened ( $25 \mathrm{Ph}$-like and 60 non-Ph-like ALL cases, Online Supplementary Table S3).

\section{Statistical analyses}

Patients' characteristics were compared by chi-squared or Fisher's exact test for categorical variables and Wilcoxon test for continuous data. Overall survival (OS), disease-free survival (DFS) and event-free survival (EFS) were estimated by the Kaplan-Meier product-limit and compared by log-rank test. OS was defined as the time between the date of diagnosis and death for any cause; patients still alive were censored at the time of the last follow-up. DFS was defined as the time between the evaluation of CR - after the induction phase - and relapse or death in $\mathrm{CR}$; patients still alive in first CR, were censored at the time of the last follow-up. Finally, EFS was defined as the time between diagnosis and non-achievement of $\mathrm{CR}$ in the induction phase, relapse or death in CR, whichever occurred first; patients still alive, in first CR, were censored at the time of the last followup.

Multivariate analysis was performed with the Cox proportional hazards regression model to adjust the effect of $B C R / A B L 1$-like predictor for clinically relevant parameters (age, white blood cell [WBC] count, hemoglobin [Hb] level, platelet count, sex and allogeneic transplant [HSCT] and for genetic aberrations impacting on prognosis [IKZF1+CDKN2A/2B and/or PAX5, K/NRAS clonal mutations, JAK/STAT clonal mutations]. ${ }^{21,22}$ All tests were 2 -sided, accepting $P<0.05$ as statistically significant. All analyses relied on the SAS v9.4 software. Study data were collected and managed using REDCap ${ }^{24}$ electronic data capture tools hosted at the GIMEMA Foundation.

\section{Results}

\section{Incidence and clinical features of Ph-like acute lymphoblastic leukemia}

We identified 28 (31.8\%) Ph-like cases with a median score of 0.85 (range, -0.18 to 6.37); the remaining 60 cases had a median score equal to -1.24 (range, -1.7 to -0.33 ). Overall, the clinical features (age, sex, WBC and platelet counts) at diagnosis of $\mathrm{Ph}$-like and of non-Ph-like cases were similar. Ph-like patients had lower $\mathrm{Hb}$ levels $(P=0.016)$, as detailed in Table 1 . The incidence of Ph-like ALL cases was slightly higher in adults ( $\geq 36$ years) than in young adults (18-35 years), being $36.2 \%$ (17 of 47 ) and $26.8 \%$ (11 of 41 ), respectively. As per clinical protocol guidelines, only $45 \%$ of $\mathrm{Ph}$-like cases were assigned to the high-risk category.

\section{Genetic features of Ph-like acute lymphoblastic leukemia cases}

The identified Ph-like cases were evaluated for the following genetic features: CRLF2 expression levels $(n=28)$, JAK/STAT and RAS pathways mutations $(n=24)$, CNA aberrations $(n=22)$ and fusion genes $(n=23)$, the latter either by RNA-sequencing and/or FISH. A CRLF2 overexpression, defined as $\Delta \mathrm{Ct}<8,{ }^{25}$ was found in 10 of $28 \mathrm{Ph}$ like cases $(35.7 \%)$. Among the CRLF2-high cases with a $\Delta \mathrm{Ct}$ value $<4.5$, we observed that three harbored a CRLF2 rearrangement, with one displaying a concomitant F232C CRLF2 mutation. Of the remaining seven CRLF2-high cases, three had a concomitant rearrangement (two ABLclass and one DDX3X/USP9X), one displayed a JAK1 and $R A S$ mutation, and in two cases the mutational screening could not be performed due to lack of genomic material; finally, in one case no additional lesions were detected. Among the $24 \mathrm{Ph}$-like cases analyzed for the mutational status, we detected a total of 13 JAK/STAT pathway mutations - nine clonal and four subclonal - in eight cases $(33.3 \%)$. Despite a high heterogeneity among samples, the most frequently mutated genes were JAK1 - affected by five mutations mainly targeting the hotspot V658 and JAK2 - affected by three mutations focused in the 


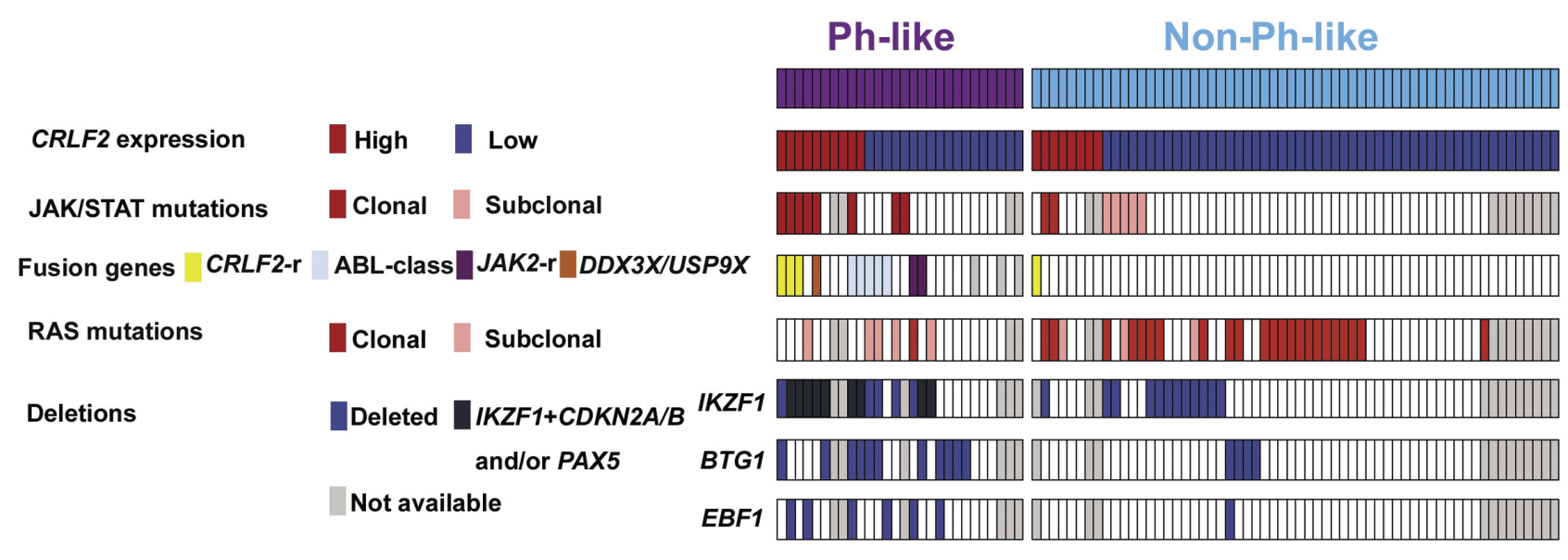

Figure 1. Distribution of the genetic lesions in the Philadelphia-like (Ph-like) and non-Ph-like cases study. Only the samples evaluated for the BCR/ABL1-like predictor and mutational status are depicted.

hotspot R683. IL7R and CRLF2 were mutated in two samples, while JAK3 only in one. Furthermore, six of the eight mutated samples $(75 \%)$ displayed a concomitant CRLF2 overexpression. Nine RAS pathway mutations - only one being clonal - were found in six patients $(25 \%)$. The most frequent mutations $(n=5)$ involved the hotspot G12-13 of $K R A S$ and NRAS. CNA analysis in Ph-like cases revealed IKZF1, BTG1, CDKN2A/2B, PAX5 and EBF1 deletions in $14(63.6 \%), 11(50 \%)$, seven (31.8\%), seven (31.8\%) and six $(27.3 \%)$ cases, respectively. Furthermore, IKZF1 + $C D K N 2 A / 2 B$ and $P A X 5$ deletions, known to confer a very poor outcome, were identified in 10 cases $(45.5 \%)$. Finally, RNA-sequencing and/or FISH experiments of the Ph-like ALL cases revealed 11 TK activating lesions (47.8\%): five ABL-class fusion genes (three NUP214/ABL1, one ZC3HAV1/ABL2 and one EBF1/PDGFRB), two BCR/JAK2, three CRLF2-r and 1 $D D X 3 X / U S P 9 X$, the latter known to be associated with CRLF2 deregulation. ${ }^{26}$

Overall, Ph-like associated lesions were identified in $70.8 \%$ (17 of 24) of cases and are summarized in Table 2.

When the genetic landscape of Ph-like ALL was compared to that of the non-Ph-like cases, significant differences emerged. As shown in Table 3, CRLF2-high was significantly more frequent in Ph-like ALL $35.7 \%$ vs. $13.3 \%, P=0.018)$. Similarly, clonal JAK/STAT mutations were specific of the Ph-like subset $(33.3 \%$ vs. $4 \%$, $P=0.001)$, while RAS pathway clonal mutations were more frequent in non-Ph-like than in Ph-like ALL cases (46\% vs. $4.2 \%, P=0.001$ ). Coincidence analysis (CNA) documented that $I K Z F 1, E B F 1$ and BTG1 deletions were significantly more common of the Ph-like than in the non-Ph-like subset $(63.6 \%, 50 \%$ and $27.3 \%$ vs. $25 \%$, $7.8 \%$ and $2.1 \%$, respectively; $P=0.002, P<0.001$ and $P=0.007) ; C D K N 2 A / 2 B$ and $P A X 5$ deletions were equally distributed among $\mathrm{Ph}$-like and non-Ph-like cases $(31.8 \%$ vs. $47.9 \%$ and $31.8 \%$ vs. $22.9 \%$, respectively).

The analysis of fusion genes, performed on a total of 85 patients, showed that rearrangements involving TK or cytokine receptors were significantly higher in the Ph-like cases with ten fusion genes involving either CRLF2 or a TK compared to only one CRLF2-r case in the non$B C R / A B L 1$-like cases $(43.5 \%$ vs. $1.6 \%, P<0.001)$.

The genetic lesions documented in both the $\mathrm{Ph}$-like and
non-Ph-like subgroups are detailed in the Online Supplementary Table S3 and their distribution is provided in Figure 1; further details on non-Ph-like ALL cases, as well as on NGS coverage, are provided in the Online Supplementary Results and Online Supplementary Table S5, respectively.

\section{Response to treatment, minimal residual disease evaluation and transplant allocation}

The Ph-like status was significantly associated with response to treatment: in fact, $\mathrm{Ph}$-like patients had a significantly inferior CR rate at time point 1 (TP1) compared to non-Ph-like cases $(74.1 \%$ vs. $91.5 \%, P=0.044$, Table 4$)$ and this translated into a lower probability of $C R$ achievement $(P=0.038, O R=0.265,95 \%$ Confidence Interval [CI]: 0.071-0.921, Online Supplementary Table S6). The latter data retained statistical significance also in a multivariate model adjusted for clinically relevant parameters, as well as for genetic lesions with a prognostic relevance.

MRD evaluation - feasible in 64 patients at TP1, 62 at TP2 and 49 at TP3 - showed that at TP1, 77.8\% of Ph-like cases and $41.3 \%$ of non-Ph-like were MRD-positive $(P=0.012)$; at TP2, $52.9 \%$ of Ph-like cases and $20 \%$ of non-Ph-like were MRD-positive $(P=0.025)$; similarly, at TP3, $41.7 \%$ of Ph-like cases and $13.5 \%$ of non-Ph-like cases were MRD-positive $(P=0.05)$. These data, summarized in Table 4, indicate that in the Ph-like patients there is a significantly higher MRD persistence at all TP evaluated compared to non-Ph-like cases. Consistently, the univariate analyses for MRD results showed that - when considering both clinically relevant parameters and genetic prognostic markers - only the Ph-like status was a risk factor for being MRD-positive at TP2 $(P=0.014, \mathrm{OR}=4.5$, 95\% CI: 1.373-15.508) (Table 5).

As a consequence, HSCT rate in first CR was significantly higher $(P=0.015)$ in $\mathrm{Ph}$-like vs. non-Ph-like cases (eight of 20 vs. 6 of $54,40 \%$ vs. $11 \%$, respectively), in line with the guidelines of the trial, in which MRD persistence was a criterion for HSCT allocation. Importantly, among five $\mathrm{MRD}+\mathrm{Ph}$-like patients who did not undergo a transplant, four relapsed at a median period a 7.8 months from CR, whereas no relapses occurred in the three $\mathrm{MRD}^{+} \mathrm{Ph}$-like patients undergoing HSCT. 
Table 2A. Genetic features of BCR/ABL1-like cases. BCR/ABL1-like BCR/ABL1 -like prediction, scoring, CRLF2 expression and mutational screening.

\begin{tabular}{|c|c|c|c|c|c|c|c|}
\hline Record ID & $\begin{array}{l}\text { BCR/ABL1-Hike } \\
\text { prediction }\end{array}$ & Score & $\begin{array}{l}\text { CRIF2 } \\
\text { expression }\end{array}$ & $\begin{array}{l}\text { RAS } \\
\text { pathway } \\
\text { status }\end{array}$ & $\begin{array}{c}\text { RAS } \\
\text { pathway } \\
\text { mutations (VAF) }\end{array}$ & $\begin{array}{l}\text { JAK/STAT } \\
\text { pathway } \\
\text { status }\end{array}$ & $\begin{array}{c}\text { JAK/STAT } \\
\text { pathway } \\
\text { mutations (VAF) }\end{array}$ \\
\hline B-ALL_1 & BCR/ABL1-like & 3.073 & Low & WT & & WT & \\
\hline B-ALL_3 & BCR/ABL1-like & 0.928 & Low & M & FLT3_ITD (5.4\%) & WT & \\
\hline B-ALL_4 & BCR/ABL1-like & 0.347 & Low & WT & & WT & \\
\hline B-ALL_7 & BCR/ABL1-like & 1.216 & High & WT & & M clonal & $\begin{array}{c}J A K 1 \text { DI630-631V (44.5\%), } \\
\text { JAKI V658I (35.5\%) }\end{array}$ \\
\hline B-ALL_16 & BCR/ABL1-like & 0.788 & Low & WT & & WT & \\
\hline B-ALL_22 & BCR/ABL1-like & 0.157 & Low & M & FLT3_V491L (11.2\%) & WT & \\
\hline B-ALL_26 & $B C R / A B L 1$-like & 3.128 & High & M & NRAS_G13D (4.1\%) & M clonal & JAK1_V658I (35.5\%) \\
\hline B-ALL_31 & BCR/ABL1-like & 2.382 & High & WT & & M clonal & CRLF2_F232C (46.8\%) \\
\hline B-ALL_32 & BCR/ABL1-like & 5.720 & Low & WT & & WT & \\
\hline B-ALL_34 & BCR/ABL1-like & 0.725 & Low & M & $\begin{array}{c}\text { PTPN11_Y279 S (1.9\%); } \\
\text { NRAS_G12D (2.6\%); } \\
\text { KRAS_G12GG (5.2\%) }\end{array}$ & WT & \\
\hline B-ALL_36 & BCR/ABL1-like & 0.205 & High & WT & & M clonal & JAK2_R683G (43.9\%) \\
\hline B-ALL_37 & $B C R / A B L 1$-like & 0.386 & Low & WT & & WT & \\
\hline B-ALL_41 & BCR/ABL1-like & 0.726 & Low & M & $\begin{array}{l}\text { KRAS_G12A (4.4\%); } \\
\text { PTPN11 V194L (4.5\%) }\end{array}$ & M clonal & $\begin{array}{l}\text { IL7R_INDEL (38.4\%); } \\
\text { JAK2_C618F (3.3\%) }\end{array}$ \\
\hline B-ALL_44 & BCR/ABLl-like & 1.587 & High & WT & & WT & \\
\hline B-ALL_45 & BCR/ABL1-like & 0.262 & Low & WT & & M clonal & $\begin{array}{c}J A K 3 \_T 21 \mathrm{M}(19.1 \%) \\
J A K 1 \_T 688 \mathrm{I}(5.7 \%)\end{array}$ \\
\hline B-ALL_46 & BCR/ABL1-like & 2.449 & Low & WT & & WT & \\
\hline B-ALL_52 & $B C R / A B L 1$-like & 1.013 & Low & WT & & WT & \\
\hline B-ALL_55 & BCR/ABL1-like & 0.544 & Low & WT & & WT & \\
\hline B-ALL_61 & BCR/ABL1-like & 2.722 & Low & NA & & $\mathrm{NA}$ & \\
\hline B-ALL_62 & BCR/ABL1-like & 0.335 & High & NA & & NA & \\
\hline B-ALL_64 & BCR/ABL1-like & -0.043 & Low & WT & & WT & \\
\hline B-ALL_73 & $B C R / A B L 1$-like & 0.048 & Low & M clonal & KRAS_G12D (35.9\%) & WT & \\
\hline B-ALL_76 & BCR/ABL1-like & 1.971 & Low & NA & & NA & \\
\hline B-ALL_81 & BCR/ABL1-like & 1.150 & High & WT & & WT & \\
\hline B-ALL_92 & BCR/ABL1-like & -0.112 & High & NA & & NA & \\
\hline B-ALL_96 & $B C R / A B L 1$-like & 6.371 & High & WT & & M clonal & CRLF2_V136M (60\%) \\
\hline B-ALL_97 & BCR/ABL1-like & 3.432 & High & WT & & M clonal & $\begin{array}{l}\text { JAK2_R683G (10.2\%); } \\
\text { IL7R_S185C (18.1\%); } \\
\text { JAK1_V658F (13.8\%) }\end{array}$ \\
\hline B-ALL_100 & BCR/ABL1-like & -0.180 & Low & WT & & WT & \\
\hline
\end{tabular}

ALL: acute lymphoblastic leukemia;VAF: variant allele frequency, FISH: fluorescence in situ hybridization; RNA seq: RNA sequencing; WT: wild-type; NA: not analzyed.

\section{Survival analyses}

Survival analyses at 24 months showed that Ph-like ALL patients had a significantly inferior EFS than non-Ph-like patients ( $33.5 \%$ vs. $66.2 \%, P=0.005)$; this difference was also evident with regard to DFS $(45.5 \%$ vs. $72.3 \%$, $P=0.062$ ), though to a lesser extent, as illustrated in Figure 2; OS was also investigated, and although not significant, it was inferior in Ph-like ALL cases than in non-Ph-like patients $(48.5 \%$ vs. $72.9 \%, P=0.16$, Online Supplementary Figure S3). The lack of significance is most likely due to the fact that a higher number of $\mathrm{Ph}$-like patients, because of persistent MRD positivity, underwent, as per protocol guidelines, HSCT ( $40 \%$ vs. $11 \%$ in Ph-like vs. non-Ph-like cases, respectively, $P=0.015$ ).

In a multivariate model for EFS, adjusting for relevant clinical parameters - including HSCT, evaluated as a time dependent covariate - and genetic prognostic markers, the $\mathrm{Ph}$-like profile, age and $\mathrm{Hb}$ levels were the only risk factors that retained statistical significance (Table 6). Notably, however, Ph-like patients undergoing an allogeneic transplant showed a trend towards better EFS $(P=0.078)$.

\section{Discussion}

The possibility of an early recognition of Ph-like ALL patients offers the unprecedented opportunity to refine the prognostic categories of $\mathrm{Ph}$-negative ALL, and to better understand the reasons for the poor outcome. In the present study, we investigated a cohort of adult B-NEG ALL patients enrolled in the front-line GIMEMA LAL1913 protocol, ${ }^{20}$ based on a pediatric-inspired backbone and in 
Table 2B. Copy number aberration (CNA) analysis, and RNA-sequencing/FISH analyses.

\begin{tabular}{|c|c|c|c|c|c|c|c|c|}
\hline Record ID & IKZF1 & CDKN2A/2B & PAX5 & $\begin{array}{c}\text { IKZF1 + CDKN2A } \\
\text { and/or PAX5 }\end{array}$ & BTG1 & EBF1 & $\begin{array}{l}\text { CDKN2A/2B } \\
\text { and/or RB1 }\end{array}$ & $\begin{array}{l}\text { Gene } \\
\text { rearrangements } \\
\text { (RNAseq and } \\
\text { or FISH analysis) }\end{array}$ \\
\hline B-ALL_1 & no- $\Delta$ & no- $\Delta$ & no- $\Delta$ & & no- $\Delta$ & $\Delta$ & no- $\Delta$ & EBF1/PDGFRB \\
\hline B-ALL_3 & $\Delta$ & $\Delta$ & $\Delta$ & Yes & no- $\Delta$ & no- $\Delta$ & $\Delta$ & No \\
\hline B-ALL_4 & no- $\Delta$ & no- $\Delta$ & no- $\Delta$ & & $\Delta$ & no- $\Delta$ & $\Delta$ & No \\
\hline B-ALL_7 & $\Delta$ & & no- $\Delta$ & Yes & no- $\Delta$ & no- $\Delta$ & $\Delta$ & DDX3X/USP9X \\
\hline B-ALL_16 & $\Delta$ & & $\Delta$ & Yes & $\Delta$ & no- $\Delta$ & $\Delta$ & BCRJJAK2 \\
\hline B-ALL_22 & $\Delta$ & no- $\Delta$ & no- $\Delta$ & & $\Delta$ & no- $\Delta$ & $\Delta$ & NUP214/ABL1 \\
\hline B-ALL_26 & $\Delta$ & no- $\Delta$ & $\Delta$ & Yes & no- $\Delta$ & & no- $\Delta$ & No \\
\hline B-ALL_31 & $\Delta$ & no- $\Delta$ & $\Delta$ & Yes & no- $\Delta$ & & no- $\Delta$ & IGH/CRLF2 \\
\hline B-ALL_32 & no- $\Delta$ & no- $\Delta$ & no- $\Delta$ & & no- $\Delta$ & no- $\Delta$ & no- $\Delta$ & NA \\
\hline B-ALL_34 & $\Delta$ & no- $\Delta$ & no- $\Delta$ & & $\Delta$ & no- $\Delta$ & no- $\Delta$ & NUP214/ABL1 \\
\hline B-ALL_36 & $\Delta$ & & no- $\Delta$ & Yes & no- $\Delta$ & no- $\Delta$ & $\Delta$ & P2RY8/CRLF2 \\
\hline B-ALL_37 & no- $\Delta$ & no- $\Delta$ & no- $\Delta$ & & $\Delta$ & & no- $\Delta$ & No \\
\hline B-ALL_41 & $\Delta$ & & no- $\Delta$ & Yes & no- $\Delta$ & no- $\Delta$ & $\Delta$ & No \\
\hline B-ALL_44A & $\Delta$ & no- $\Delta$ & $\Delta$ & Yes & $\Delta$ & no- $\Delta$ & $\Delta$ & ZC3HAVI/ABL2 \\
\hline B-ALL_45 & $\mathrm{NA}$ & NA & NA & & $\mathrm{NA}$ & NA & NA & No \\
\hline B-ALL_46 & no- $\Delta$ & no- $\Delta$ & no- $\Delta$ & & no- $\Delta$ & no- $\Delta$ & no- $\Delta$ & No \\
\hline B-ALL_52 & no- $\Delta$ & $\Delta$ & $\Delta$ & & $\Delta$ & no- $\Delta$ & $\Delta$ & No \\
\hline B-ALL_55 & no- $\Delta$ & no- $\Delta$ & no- $\Delta$ & & $\Delta$ & no- $\Delta$ & no- $\Delta$ & No \\
\hline B-ALL_61 & $\mathrm{NA}$ & $\mathrm{NA}$ & $\mathrm{NA}$ & & $\mathrm{NA}$ & NA & NA & No \\
\hline B-ALL_62 & NA & NA & NA & & NA & NA & $\mathrm{NA}$ & No \\
\hline B-ALL_64 & NA & $\mathrm{NA}$ & $\mathrm{NA}$ & & $\mathrm{NA}$ & $\mathrm{NA}$ & $\mathrm{NA}$ & NA \\
\hline B-ALL_73 & $\Delta$ & no- $\Delta$ & no- $\Delta$ & & no- $\Delta$ & $\Delta$ & no- $\Delta$ & BCRJJAK2 \\
\hline B-ALL_76 & NA & NA & NA & & NA & NA & NA & NA \\
\hline B-ALL_81 & $\Delta$ & $\Delta$ & no- $\Delta$ & Yes & $\Delta$ & no- & $\Delta$ & No \\
\hline B-ALL_92 & $\mathrm{NA}$ & $\mathrm{NA}$ & $\mathrm{NA}$ & & NA & NA & $\mathrm{NA}$ & No \\
\hline B-ALL_96 & $\Delta$ & no- $\Delta$ & $\Delta$ & Yes & $\Delta$ & $\Delta$ & no- $\Delta$ & NUP214/ABL1 \\
\hline B-ALL_97 & $\Delta$ & no- $\Delta$ & no- $\Delta$ & & $\Delta$ & no- $\Delta$ & no- $\Delta$ & IGH/CRLF2 \\
\hline B-ALL_100 & no- $\Delta$ & no- $\Delta$ & no- $\Delta$ & & no- $\Delta$ & no- $\Delta$ & no- $\Delta$ & No \\
\hline
\end{tabular}

which MRD quantification at week 10 is pivotal for transplant allocation, in order to assess the prognostic impact of the Ph-like status. In particular, we aimed at understanding the interplay between the Ph-like status and MRD response. Furthermore, we sought to analyze the clinical and genetic features, the hematologic responses to treatment and the outcome of the identified Ph-like ALL patients.

The screening carried out using the BCR/ABL1-like predictor $^{7}$ led to the identification of $28 \mathrm{Ph}$-like cases - representing $31.8 \%$ of the B-NEG cohort - with a slightly higher incidence in adults than in young adults. This finding is in agreement with the recently reported data in other adult cohorts and resembles the epidemiologic behavior of "true Ph-positive" ALL. 5,6,19 The comparison of the clinicalbiological features of $\mathrm{Ph}$-like and non-Ph-like cases revealed a substantial homogeneity in terms of WBC count and sex distribution, as in the GMALL and the MDACC clinical trials, ${ }^{6,19}$ and at variance from Roberts and colleagues ${ }^{5}$ who reported that adult $B C R / A B L 1$-like patients have a higher WBC and are prevalently of male sex. In children, an association with hyperleukocyotsis has been described by Den Boer et al. ${ }^{1}$ and Reshmi et al., ${ }^{27}$ the latter based on the COG AALL1131 high-risk cohort.
The association with male sex was documented in the Total Therapy XV cohort. ${ }^{16}$ On the contrary, Roberts and colleagues ${ }^{28}$ did not find significant differences in the WBC count and sex in the standard-risk subset of childhood B-ALL patients enrolled in the COG AALL0331. In addition to the WBC count and sex, it is worth underlying that in our study the population of Ph-like patients was allocated to both the standard- (56\%) and high-risk (44\%) categories: this finding has important clinical implications since the prompt identification of these cases might lead to a better therapeutic stratification that ultimately would avoid undertreating these high-risk patients. In adults, a similar distribution was reported also by Herold et al., while in the pediatric setting this issue is still controversial. Indeed, most Ph-like cases were associated to a high risk in both the COALL and DCOG cohorts, ${ }^{1}$ while in the Total Therapy XV trial16 Ph-like cases were equally distributed in the standard and high National Cancer Institute (NCI) risk groups. Of note, in the report on 139 children classified as standard-risk, Roberts and colleagues ${ }^{28}$ showed that the Ph-like status did not affect outcome, suggesting that in children risk stratification is clinically more significant than the genomic features.

From a genetic standpoint, the present study further cor- 
Table 3. Comparison between Philadelphia-like (Ph-like) and non-Ph-like genetic features.

\begin{tabular}{|c|c|c|c|}
\hline & $B C R / A B L 1-H K e$ & nOn-BCR/ABL1-Hke & $P$ \\
\hline \multicolumn{4}{|l|}{ CRLF2 expression level } \\
\hline CRLF2 overexpressing samples & $10 / 28(35.7 \%)$ & $8 / 60(13.3 \%)$ & 0.018 \\
\hline \multicolumn{4}{|l|}{ Mutational status } \\
\hline RAS pathway mutated samples & $6 / 24(25 \%)$ & $26 / 50(52 \%)$ & 0.025 \\
\hline Clonal RAS mutated & $1 / 24(4.16 \%)$ & $23 / 50(46 \%)$ & 0.001 \\
\hline JAK/STAT pathway mutated samples & $8 / 24(33.3 \%)$ & $7 / 50(14 \%)$ & 0.04 \\
\hline Clonal JAK/STAT mutated & $8 / 24(33.3 \%)$ & $2 / 50(4 \%)$ & 0.001 \\
\hline \multicolumn{4}{|l|}{ Copy number aberrations } \\
\hline$I K Z F 1$ deleted & $14 / 22(63.6 \%)$ & $12 / 48(25 \%)$ & 0.002 \\
\hline$I K Z F 1+C D K N 2 A / 2 B$ and/or $P A X 5$ & $10 / 22(45.5 \%)$ & $7 / 48(14.6 \%)$ & 0.007 \\
\hline BTG1 deleted & $11 / 22(50 \%)$ & $4 / 48(8.3 \%)$ & $<0.001$ \\
\hline EBFl deleted & 6/22 (27.3\%) & $1 / 48(2.1 \%)$ & 0.003 \\
\hline$C D K N 2 A 2 B$ deleted & $7 / 22(31.8 \%)$ & $23 / 48(47.9 \%)$ & ns \\
\hline PAX5 deleted & $7 / 22(31.8 \%)$ & $11 / 48(22.9 \%)$ & ns \\
\hline TK or cytokine receptor fusion genes & $10 / 23(43.5 \%)$ & $1 / 37(2.7 \%)$ & $<0.001$ \\
\hline
\end{tabular}

TK: tyrosine kinase; ns: not significant.

Table 4. Complete remission achievement and minimal residual disease evaluation in Philadelphia-like (Ph-like) and non-Ph-like cases.

\begin{tabular}{|c|c|c|c|}
\hline & Ph-llke & non-Ph-llke & $P$ \\
\hline CR achievement & $20(74.1 \%)$ & $54(91.5 \%)$ & 0.044 \\
\hline \multicolumn{4}{|l|}{ TP1 (week 4) } \\
\hline MRD-positive patients & 14/18 (77.8\%) & $19 / 46(41.3 \%)$ & 0.012 \\
\hline \multicolumn{4}{|l|}{ TP2 (week 10) } \\
\hline MRD-positive patients & $9 / 17(52.9 \%)$ & $9 / 45(20 \%)$ & 0.025 \\
\hline \multicolumn{4}{|l|}{ TP3 (week 16) } \\
\hline MRD-positive patients & $5 / 12(41.7 \%)$ & 5/37 (13.5\%) & 0.05 \\
\hline
\end{tabular}

Ph-like: Philadelphia-like; CR: complete remission; TP: time point; MRD: minimal residual disease.

Table 5. Univariate analyses for minimal residual disease at time point 2, considering clinically relevant variables and molecular prognostic markers.

\begin{tabular}{lcc} 
& Univariate antilysis for MRiD_IP2 & $P$ \\
Ph-like $v$ s. non-Ph-like & OR (95\%Cl) & 0.014 \\
Age & $4.5(1.373-15.508)$ & 0.475 \\
WBC & $1.012(0.98-1.045)$ & 0.133 \\
Plts & $1.013(1-1.033)$ & 0.0365 \\
Hb & $0.987(0.974-0.998)$ & 0.152 \\
F vs. M & $0.832(0.638-1.06)$ & 0.1602 \\
No SR vs.SR & $0.459(0.145-1.315)$ & 0.083 \\
IKZFI+ CDKN2A2B and/or PAX5 vs IKZF1-only/WT & $0.304(0.065-1.048)$ & 0.339 \\
Cell cycle genes deletion vs WT & $1.869(0.49-6.674)$ & 0.8253 \\
RAS clonal vs WT/M subclonal & $0.88(0.279-2.773)$ & 0.706 \\
JAK/STAT clonal vs WT/M subclonal & $0.8(0.239-2.51)$ & 0.2482 \\
\hline
\end{tabular}

MRD: minimal residual disease;Ph-like:Philadelphia-like;WBC: white blood cell; Plt:platelet;Hb: hemoglobin;F: female; M: male;SR: standard risk;WT: wild-type;WT/M: wild-type/ mutated; OR: odds ratio; CI: Confidence Interval.

roborates the notion that CRLF2 overexpression, JAK/STAT mutations and deletions of IKZF1, BTG1 and EBF1 are significantly more frequent in Ph-like ALL cases. In addition, we observed that clonal JAK/STAT mutations were almost exclusively found in Ph-like ALL, while clonal RAS mutations were specific of non-Ph-like cases, thus suggesting that they play a different role in the two molecular subtypes. Moreover, when focusing on CRLF2 overexpression, it emerges that it is not sufficient to induce a Ph-like profile: indeed, of the eight Ph-like cases that were fully characterized, seven had at least another lesion.
Furthermore, the results on the incidence of rearrangements targeting TK and cytokine receptors indicate that they prevail in the Ph-like subgroup, with $\mathrm{ABL}$-class gene rearrangements outnumbering the other lesions. Thus, we could identify at least one underlying genetic lesion in $70.8 \%$ of Ph-like patients. Not for all cases was it possible to perform an extensive biological screening due to the lack of genomic material (four cases) and RNA-sequencing was carried out using targeted approaches and not genomewide tools. This may help to explain why no further genetic lesions could be found in the remaining cases (29.2\%) 
Table 6. Summary of univariate and multivariate analyses for event-free survival, considering clinically relevant variables and molecular prognostic markers.

\begin{tabular}{|c|c|c|c|c|}
\hline & \multicolumn{2}{|c|}{ Univariate analysis for EFS } & \multicolumn{2}{|c|}{ Multivariate analysis for EFS } \\
\hline & HR (95\% Cl) & $P$ & HR (95\% Cl) & $P$ \\
\hline Ph-like $v s$. non-Ph-like & $2.6(1.3-5.19)$ & 0.007 & $2.3(1.124-4.92)$ & 0.023 \\
\hline Age & $1.03(1.01-1.05)$ & 0.004 & $1.04(1.015-1.067)$ & 0.002 \\
\hline WBC & $1.005(0.999-1.010)$ & 0.074 & & \\
\hline Plt & $0.993(0.986-0.999)$ & 0.023 & & \\
\hline $\mathrm{Hb}$ & $0.81(0.69-0.94)$ & 0.006 & $0.782(0.649-0.943)$ & 0.01 \\
\hline Fus. M & $0.78(0.41-1.5)$ & 0.455 & & \\
\hline No SR vs. SR & $1.89(0.97-3.67)$ & 0.062 & & \\
\hline $\begin{array}{l}\text { HSCT } v \text { s. No HSCT as a time } \\
\text { dependendent covariate }\end{array}$ & $1.04(0.35-3.10)$ & 0.939 & & \\
\hline $\begin{array}{l}I K Z F 1+C D K N 2 A 2 B \text { and/or } \\
P A X 5 \text { vs. IKZF1-only/WT }\end{array}$ & $1.73(0.76-3.98)$ & 0.193 & & \\
\hline Cell cycle genes deletion $v s$. WT & $0.967(0.451-2.069)$ & 0.93 & & \\
\hline RAS clonal vs. WT/M subclonal & $0.604(0.269-1.358)$ & 0.222 & & \\
\hline JAK/STAT clonal vs. WT/M subclonal & $0.85(0.26-2.82)$ & 0.796 & & \\
\hline
\end{tabular}

EFS: event-free survival; Ph-like: Philadelphia-like;WBC: white blood cell; Plt: platelet; Hb: hemoglobin; F: female; M: male; SR: standard risk; WT: wild-type; HSCT: allogeneic stem cell transplant;WT/M: wild-type/mutated; OR: odds ratio; CI: Confidence Interval.
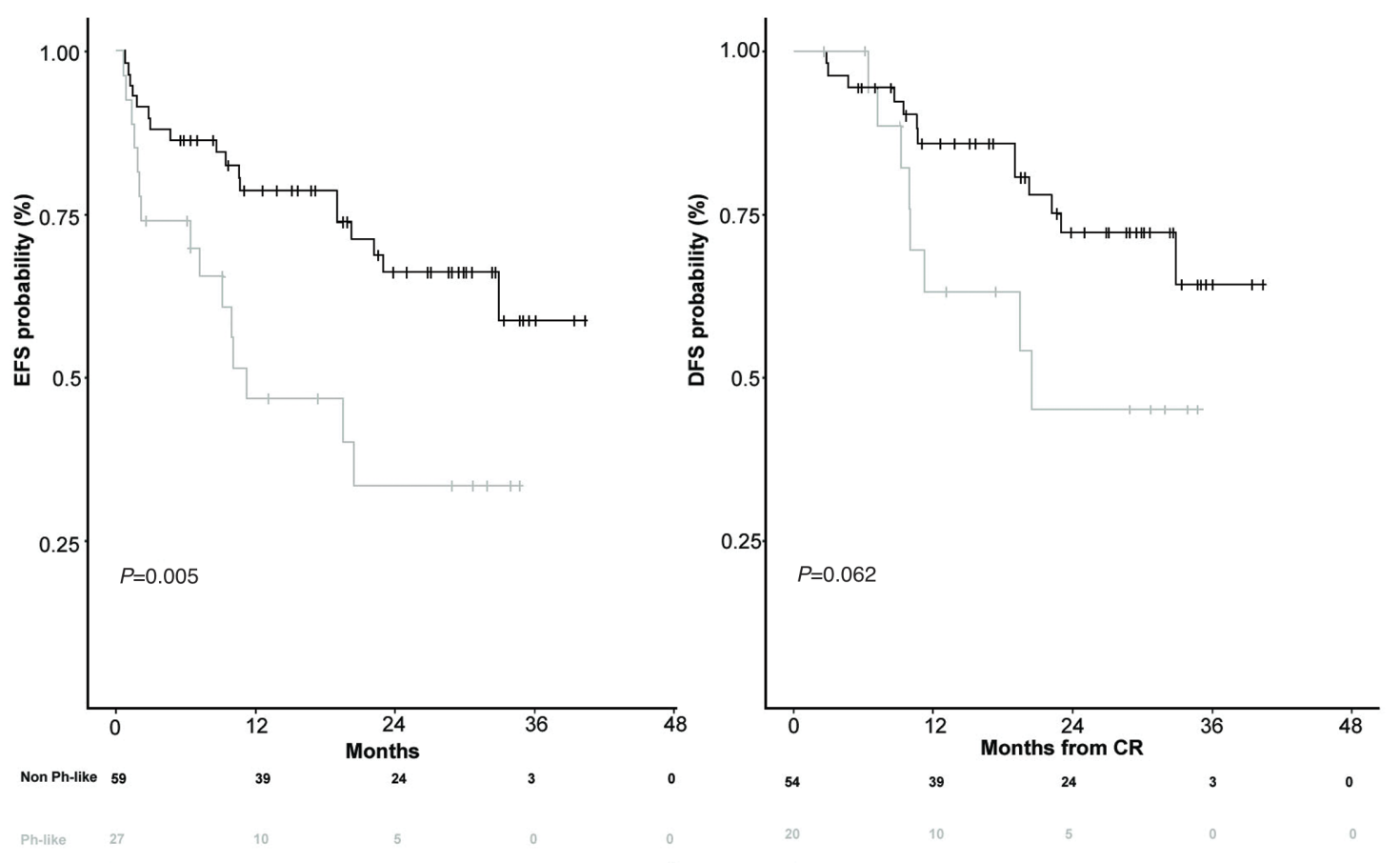

Figure 2. Survival curves of Philadelphia-like (Ph-like) and non-Ph-like patients. event-free survival and disease-free survival.

that proved positive with the BCR/ABL1 predictor. The validity and reproducibility of the $B C R$ - $A B L 1$-like predictor has been externally validated by other institutions and from external samples in Europe, showing an overall concordance with other tools (FISH and NGS) of $88 \%{ }^{29}$

With regards to the relationship between the Ph-like status, $\mathrm{MRD}$ response and outcome, we showed that $\mathrm{Ph}$-like ALL patients have a higher risk of CR failure: in fact, $74.1 \%$ of Ph-like ALL and $91.4 \%$ non-Ph-like achieved a $\mathrm{CR}$. This difference was neither detected in the intensive GMALL trials $06 / 99$ and $07 / 03$ - in which all patients achieved a CR, albeit with a short duration,$-{ }^{6}$ nor in the
hyper-CVAD-based protocols or the augmented BFM regimen administered at MDACC. ${ }^{19}$

More importantly, our study allowed to correlate the $\mathrm{Ph}$-like status with $\mathrm{MRD}$, that is presently regarded as the most important prognostic marker in ALL management. In fact, this analysis showed that in the GIMEMA LAL1913 protocol, at all TP analyzed, the percentage of MRD-positive patients was significantly higher in the Phlike ALL subset than in non-Ph-like cases. This difference was particularly evident at TP2 (HSCT decisional point), when $52.9 \%$ of Ph-like and only $20 \%$ of non-Ph-like cases were MRD-positive. Indeed, when both clinically relevant 
parameters and genetic prognostic markers were taken into account the $\mathrm{Ph}$-like profile proved the only risk factor for MRD positivity at TP2. Thus, considering both response to induction treatment and $\mathrm{MRD}$ monitoring, the Ph-like status, if identified early, permits not only to recognize patients who are likely to be refractory to induction treatment, but also to identify - within cases who achieve a CR - those who are likely to remain MRD-positive. This strong association may allow to anticipate therapeutic changes.

To our knowledge, this is the first study that analyzes the interaction between the Ph-like status and MRD assessed by quantitative PCR of the IG and TR gene rearrangements - in a broad cohort of uniformly and prospectively treated adult ALL patients within a clinical trial. Similar results were provided by Herold and colleagues ${ }^{6}$ who found that Ph-like patients were less likely to achieve a MRD-negative status in a small cohort of 31 patients with overlapping $\mathrm{MRD}$ and Ph-like status information. In the pediatric setting, contradicting results have been reported. ${ }^{14,16}$

Furthermore, the comparison of survival curves highlighted that $\mathrm{Ph}$-like patients experienced a significantly worse EFS at 24 months compared to that of non-Ph-like cases $(33.5 \%$ and $66.2 \%$, respectively). Along the same line, also in cases achieving a CR, the Ph-like profile had a negative prognostic impact, as shown by the worse DFS of Ph-like patients. Although limited by the small sample size, our study demonstrates that transplant is beneficial in these cases and should be pursued at the earliest opportunity, as shown by the high rate of relapses within nontransplanted Ph-like patients (4 of $5 \mathrm{MRD}$ positive patients relapsed).

Lastly, in all outcome parameters evaluated - CR achievement, MRD at TP2 and EFS - the Ph-like status emerged as an independent prognostic marker.

In addition to confirming the inferior outcome of Ph-like ALL patients, these data indicate that the differences between $\mathrm{Ph}$-like and non-Ph-like cases are not abolished by pediatric-like intensive therapeutic schemes, in agreement with the results of the MDACC group. ${ }^{18}$ Based on the MRD findings hereby reported, this is primarily contributed to the significantly lower rates of complete molecular responses observed in Ph-like patients.

In light of the poor outcome of Ph-like ALL and of the possibility of using targeted approaches, ${ }^{30}$ different clinical trials specifically designed for $\mathrm{Ph}^{+} \mathrm{ALL}$ and $\mathrm{Ph}$-like ALL cases are testing the efficacy of dasatinib (clinicaltrials gov. Identifier: 02420717, 02883049, 03564470 and 02143414) or of dasatinib in combination with blinatumomab (clinicaltrials gov. Identifier: SWOG-S1318 and NCT02143414). Other studies are investigating the impact of blinatumomab in combination with chemotherapy in Ph-negative B-lineage ALL (GIMEMA LAL2317, clinicaltrials gov. Identifier: 03367299 and 02003222). In these latter studies, it is investigated if the addition of blinatumomab can increase the rates of $\mathrm{CR}$ and MRD-negativity in Ph-like patients, as already observed in $\mathrm{Ph}^{+}$ALL. $^{32}$ In support of the fact that Ph-like patients may benefit from targeted treatment, a recent study from Tanasi and colleagues has reported that the introduction of TKI front-line was associated with a 3 -years OS of $77 \% .{ }^{31}$ Other compounds, such as ruxolitinib (clinicaltrials gov. Identifier: 02420717, 03571321 and 02723994) and the histone deacetylase inhibitor chidamide (clinicaltrials gov. Identifier: 03564470) are under investigation.

Taken together, the results of this study carried out on adult B-NEG ALL cases enrolled in the front-line GIMEMA LAL1913 clinical protocol confirm that the BCR/ABL1-like predictor ${ }^{7}$ is a valid tool to rapidly recognize Ph-like cases that account for about $30 \%$ of adult B-NEG ALL. In addition, we could show that also in a pediatric-oriented and MRD-driven clinical trial $\mathrm{Ph}$-like patients have a lower probability of achieving a CR, are more likely to remain MRD-positive and have a significantly shorter EFS. The Ph-like profile is an independent risk factor for $\mathrm{CR}$ failure and MRD-persistence, thus further underlying the need that Ph-like cases - a primary unmet clinical need in ALL - are rapidly recognized at diagnosis in order to refine the risk stratification of $\mathrm{Ph}$ negative ALL and optimize patients' management. Further investigations are currently ongoing to unravel if within $\mathrm{Ph}$-like ALL there are subgroups of patients with a different outcome likelihood.

\section{Disclosures}

No conflicts of interest to disclose.

\section{Contributions}

SC designed research, analyzed data, provided clinical samples and clinical data, and wrote the manuscript; MM performed experiments, analyzed data and wrote the manuscript; $A P$ performed statistical analyses; IDS, LC, AT, MC, LE, GAP, RLS, MCAL, MCP, VP, AS, OS, VA performed experiments; SC, $F D R, P D F, C P, A C, R C, M C, N F, D M, C C, A V$, provided samples and clinical data; EC and PF contributed to protocol management; $A G$ and $C M$ critically revised the manuscript; $A R$ and $R B$ designed the trial and critically revised the manuscript; $R F$ designed the research and the trial, and critically revised the manuscript.

\section{Acknowledgments}

The authors wish to thank Grazia Fazio and Chiara Palmi for support in TruSight Pancancer and MLPA experiments, Associazione Italiana per la Ricerca sul Cancro (AIRC) 5×1000, Special Program Metastases (21198), Milan (Italy) to RF; Finanziamento Medi Progetti Universitari 2015 to SC (Sapienza University of Rome); Bandi di Ateneo per la Ricerca (Sapienza University of Rome, RM11816436B712AF) to SC and PRIN 2017 (2017PPS2X4_002) to SC, CM, and RLS; Fondazione Cassa di Risparmio di Perugia (grant number 2018.0418.021) to RLS.

\section{References}

1. Den Boer ML, van Slegtenhorst M, De Menezes RX, et al. A subtype of childhood acute lymphoblastic leukaemia with poor treatment outcome: a genome-wide classifi- cation study. Lancet Oncol. 2009;10(2):125134.

2. Mullighan CG, Su X, Zhang J, et al. Deletion of IKZF1 and prognosis in acute lymphoblastic leukemia. N Engl J Med. 2009; 360(5):470-480

3. Roberts KG, Morin RD, Zhang J, et al.
Genetic alterations activating kinase and cytokine receptor signaling in high-risk acute kymphoblastic leukemia. Cancer Cell. 2012;22(2):153-166.

4. Roberts KG, Li Y, Payne-Turner D, et al. Targetable kinase-activating lesions in Ph-like acute lymphoblastic leukemia. N 
Engl J Med. 2014;371(11):1005-1015.

5. Roberts KG, Gu Z, Payne-Turner D, et al. High frequency and poor outcome of Philadelphia chromosome-like acute lymphoblastic leukemia in adults. J Clin Oncol. 2017;35(4):394-401.

6. Herold T, Schneider S, Metzeler KH, et al. Adults with philadelphia chromosome-like acute lymphoblastic leukemia frequently have igh-CRLF2 and JAK2 mutations, persistence of minimal residual disease and poor prognosis. Haematologica. 2017; 102(1):130-138.

7. Chiaretti S, Messina M, Grammatico S, et al. Rapid identification of BCR/ABL1-like acute lymphoblastic leukaemia patients using a predictive statistical model based on quantitative real time-polymerase chain reaction: clinical, prognostic and therapeutic implications. Br J Haematol. 2018;181(5):642-652.

8. Maude SL, Tasian SK, Vincent T, et al. Targeting JAK1/2 and mTOR in murine xenograft models of Ph-like acute lymphoblastic leukemia. Blood. 2012; 120(17):3510-3518.

9. Lengline $\mathrm{E}$, Beldjord $\mathrm{K}$, Dombret $\mathrm{H}$, et al. Successful tyrosine kinase inhibitor therapy in a refractory B-cell precursor acute lymphoblastic leukemia with EBF1-PDGFRB fusion. Haematologica. 2013;98(11):146-148.

10. Weston BW, Hayden MA, Roberts KG, et al. Tyrosine kinase inhibitor therapy induces remission in a patient with refractory EBF1PDGFRB-positive acute lymphoblastic leukemia. J Clin Oncol. 2013;31(25):e413416.

11. Fazio F, Barberi W, Cazzaniga G, et al. Efficacy of imatinib and chemotherapy in a pediatric patient with Philadelphia-like acute lymphoblastic leukemia with EBF1PDGFRB fusion transcript. Leuk Lymphoma. 2020;61(2):469-472.

12. Tasian SK, Teachey DT, Li Y, et al. Potent efficacy of combined PIBK/mTOR and JAK or ABL inhibition in murine xenograft models of Ph-like acute lymphoblastic leukemia. Blood. 2017;129(2):177-187.

13. Harvey RC, Kang H, Roberts KG, et al. Development and validation of a highly sensitive and specific gene expression classifier to prospectively screen and identify B-pre- cursor acute lymphoblastic leukemia (ALL) patients with a Philadelphia chromosomelike ("Ph-like" or "BCR-ABL1-Like") signature for therapeutic targeting and clinical intervention. Blood. 2013;122(21):826.

14. Heatley SL, Sadras T, Kok CH, et al. High prevalence of relapse in children with Philadelphia-like acute lymphoblastic leukemia despite risk-adapted treatment. Haematologica. 2017;102(12):e490-e493.

15. Roberts KG. The biology of Philadelphia chromosome-like ALL. Best Pract Res Clin Haematol. 2017;30(3):212-221.

16. Roberts KG, Pei D, Campana D, et al. Outcomes of children with BCR-ABL1-like acute lymphoblastic leukemia treated with risk-directed therapy based on the levels of minimal residual disease. J Clin Oncol. 2014;32(27):3012-3020.

17. Stock W, Luger SM, Advani AS, et al. A pediatric regimen for older adolescents and young adults with acute lymphoblastic leukemia: results of CALGB 10403. Blood. 2019;133(14):1548-1559.

18. Tasian SK, Hurtz C, Wertheim GB, et al. High incidence of Philadelphia chromosome-like acute lymphoblastic leukemia in older adults with B-ALL. Leukemia. 2017; 31(4):981-984.

19. Jain N, Roberts KG, Jabbour E, et al. Ph-like acute lymphoblastic leukemia: a high-risk subtype in adults. Blood. 2017;129(5):572581.

20. Bassan R, Chiaretti S, Paoloni F, et al. First results of the GIMEMA LAL1913 protocol for adult patients with Philadelphia-negative acute lymphoblastic leukemia (Ph- ALL). On behalf of the GIMEMA Acute Leukemia Working Group. PS919. HemaSphere. 2018;2(S1):408.

21. Messina M, Chiaretti S, Wang J, et al. Prognostic and therapeutic role of targetable lesions in B-lineage acute lymphoblastic leukemia without recurrent fusion genes. Oncotarget. 2016;7(12):13886-13901.

21. Messina M, Chiaretti S, Fedullo AL, et al. Clinical significance of recurrent copy number aberrations in B-lineage acute lymphoblastic leukaemia without recurrent fusion genes across age cohorts. Br J Haematol. 2017;178(4):583-587.
23. Fedullo AL, Messina M, Elia L, et al Prognostic implications of additional genomic lesions in adult $\mathrm{Ph}+$ acute $\mathrm{lym}$ phoblastic leukemia. Haematologica. 2019; 104(2):312-318.

24. Harris PA, Taylor R, Thielke R, Payne J, Gonzalez N, Conde JG. Research electronic data capture (REDCap). A metadata-driven methodology and workflow process for providing translational research informatics support. J Biomed Inform. 2009;42(2):377-381.

25. Chiaretti S, Brugnoletti F, Messina M, et al. CRLF2 overexpression identifies an unfavourable subgroup of adult B-cell precursor acute lymphoblastic leukemia lacking recurrent genetic abnormalities. Leuk Res. 2016;41:36-42.

26. Russell LJ, Jones L, Enshaei A, et al Characterisation of the genomic landscape of CRLF2-rearranged acute lymphoblastic leukemia. Genes Chromosomes Cancer. 2017;56(5):363-372.

27. Reshmi SC, Harvey RC, Roberts KG, et al. Targetable kinase gene fusions in high-risk B-ALL: A study from the Children's Oncology Group. Blood. 2017;129(25):33523361.

28. Roberts KG, Reshmi SC, Harvey RC, et al. Genomic and outcome analyses of Ph-like ALL in NCI standard-risk patients: a report from the children's oncology group. Blood. 2018;132(8):815-824.

29. Chiaretti S, Taherinasab A, Canichella M, et al. The Validation of the BCR/ABL1-like predictor across laboratories shows reproducibility of results. Blood. 2019: 134 (Suppl 1):S5211.

30. Chiaretti S, Messina M, Foà R. BCR/ABL1like acute lymphoblastic leukemia: how to diagnose and treat? Cancer. 2019;125(2): 194-204.

31. Tanasi I, Ba I, Sirvent N, et al. Efficacy of tyrosine kinase inhibitors in Ph-like acute lymphoblastic leukemia harboring ABLclass rearrangements. Blood. 2019;134(16): 1351-1355.

32. Foà R, Bassan R, Vitale A, et al. GIMEMA Investigators. Dasatinib-nlinatumomab for $\mathrm{Ph}$-positive acute lymphoblastic leukemia in adults. N Engl J Med. 2020;383(17):16131623 